\title{
À PROPOS DU COLLOQUE « THE MACHINE AS MODEL AND METAPHOR » Max-Planck-Institut für Wissenschaftsgeschichte Berlin, novembre 2006
}

\begin{abstract}
Sophie Roux*
«Lève la peau, dissèque: ici commencent les machines. Puis, tu te perds dans une substance inexplicable, étrangère à tout ce que tu sais et qui est pourtant l'essentielle. »
\end{abstract}

Paul Valéry, Tel quel, Cahier B 1910

Le colloque « The Machine as Model and Metaphor », qui a eu lieu au Max-PlanckInstitut für Wissenschaftsgeschichte (Berlin) en novembre 2006 était le quatrième colloque de l'équipe 1 du programme « From Natural Philosophy to Science », financé par la Fondation européenne pour la science ${ }^{1}$. L'objet de ce colloque était très généralement de déterminer dans quelle mesure les machines ont, du XIII ${ }^{\mathrm{e}}$ au XVII ${ }^{\mathrm{e}}$ siècle, contribué à reconfigurer un certain nombre d'idées en ce qui concerne la manière dont les phénomènes naturels peuvent ou doivent être expliqués. Le problème n'était donc pas tant de déterminer comment philosophes naturels, ingénieurs et mathématiciens procédèrent pour identifier les principes mécaniques selon lesquels les machines fonctionnent que de se demander dans quelle mesure ils en vinrent à considérer les

\footnotetext{
${ }^{*}$ Sophie Roux est maître de conférences à l'université de Grenoble II/Institut universitaire de France (Université Pierre Mendès France, UFR Sciences Humaines, BP 47, 1281, avenue centrale, F-38040 Grenoble cedex 9; Sophie.Roux@upmf-grenoble.fr). Ses recherches portent sur l'épistémologie, l'histoire des sciences et l'histoire de la philosophie à l'âge classique (XVI ${ }^{\mathrm{e}}-\mathrm{XVII}^{\mathrm{e}}$ siècles). Elle a notamment dirigé avec Walter Roy Laird, Mechanics and Natural Philosophy before the Scientific Revolution (Dortrecht/Boston/Londres, Kluwer Academic Publishers, 2007), puis, en collaboration avec Massimo Bucciantini et Michele Camerota, Mechanics and Cosmology in the Medieval and Early Modern Period (Florence, Olschki, 2007).

1. Ce colloque était organisé conjointement par Jochen Büttner et Sophie Roux. Pour une présentation de l'ensemble de ce programme, voir les sites http:/www.ru.nl/filosofie/center/esf/ et http:/www.esf.org/activities/research-networking-programmes/humanities-sch/current-esf-researchnetworking-programmes-in-humanities/from-natural-philosophy-to-science.html.
} 
machines comme des modèles d'intelligibilité. Autrement dit, au lieu de faire des machines des explananda, comme on le fait usuellement en histoire de la mécanique, il s'agissait de les examiner en tant qu'explanantia. Les deux questions ne sont toutefois pas mutuellement exclusives. Dire que les machines sont des explanantia n'implique pas que l'enquête physique s'arrête une fois qu'on a montré que les phénomènes naturels examinés fonctionnent comme des machines; cela peut au contraire susciter de nouvelles questions.

Les références à des mécanismes particuliers ou aux machines en général abondent dans les textes de la Renaissance et de l'âge classique, en particulier dans les textes de sciences et de philosophie, mais pas seulement. Les références aux machines semblent cependant favoriser l'équivocité plutôt que tendre à l'univocité ${ }^{2}$. C'est que, étant donné la diversité des idées qu'elle recouvre, l'analogie entre phénomènes naturels et machines est une analogie plus ouverte que contraignante. En premier lieu, comme l'ont montré plusieurs travaux récents, la signification du terme « machine » change à la période considérée ${ }^{3}$. Durant l'Antiquité et au Moyen Âge, le terme «machina» se réfère principalement à de grosses structures stables, en particulier des échafaudages, des appareils de levage, et certaines machines de guerre comme les tours d'assaut. À la Renaissance, des auteurs comme Georgius Agricola, Daniele Barbaro ou Alessandro Giorgi, en quête de reconnaissance sociale, empruntèrent le terme « machina » à l'autorité classique qu'était Vitruve pour donner une unité au moins nominale aux différents dispositifs mécaniques qu'ils mettaient en œuvre. Mais ce terme, dont le succès est confirmé par les Théâtres de machines d'Agostino Ramelli, de Jacques Besson ou de Salomon de Caus, a alors changé de référent: il ne se rapporte plus à de grosses structures stables, mais à des dispositifs complexes capables de fonctionner continument avec une relative autonomie et d'accomplir un certain travail, comme par exemple les moulins ou les systèmes d'engrenages. Conformément à la célèbre analyse de Marx, ces dispositifs peuvent être décomposés en trois parties qui correspondent à trois fonctions : le moteur, la transmission du mouvement, l'instrument ou l'outil qui accomplit le travail à proprement parler ${ }^{4}$.

En deuxième lieu, même si l'on restreint l'analyse à ce qu'on pourrait appeler les « machines marxistes », ce ne sont pas toujours les mêmes caractéristiques des machines, ou pas toujours les mêmes machines, qui autorisent une analogie avec les phénomènes naturels ${ }^{5}$. Il peut s'agir de souligner qu'un phénomène a un fonctionnement comparable à celui d'un dispositif artificiel: on fait alors fonds sur l'opposition entre le naturel et l'artificiel, le mécanique et le machinique étant employés synecdotiquement pour les techniques en général. On peut aussi s'appuyer sur une caractéristique assez récente à l'époque des machines en général et des horloges en particulier, leur fonctionnement régulier, pour insister sur le caractère ordonné et pour tout dire légal des phénomènes

2. Sur cette équivocité, voir également VenEZIANI, 2005.

3. Dans ce paragraphe, je résume Popplow, 2007, p. 48-60. Voir également les références données infra note 15 .

4. MARX, 1867, ici 1965, liv. I, IV e sect., chap. Xv, § 1, p. 916 sqq.

5. Je me restreins aux usages scientifiques de cette analogie; ses usages sociaux et politiques ont été analysés de manière stimulante dans deux ouvrages bien différents de facture: MAYR, 1986; Schlanger, 1971. 
naturels. Si maintenant on pense plus spécifiquement aux machines automates, ce qui intervient, c'est leur capacité à se mouvoir d'elles-mêmes. Enfin, le machinique peut ne pas être bien loin du mécanique, lorsqu'on dit qu'un phénomène est analogue à une machine parce qu'il accomplit ses fonctions par la seule combinaison des parties matérielles qui le composent, une fois celles-ci mises en mouvement.

En troisième lieu, les comparaisons entre phénomènes naturels et machines s'accompagnent d'engagements ontologiques variables, allant d'un usage d'analogies limitées et superficielles jusqu'à la thèse réductionniste que tous les phénomènes naturels peuvent être réduits à des dispositifs mécaniques. L'appréhension des animaux constitue un cas exemplaire de cette variabilité ontologique: déjà dans l'Antiquité, et particulièrement chez Galien, on trouve des analogies entre le fonctionnement des organes animaux et le fonctionnement de dispositifs artificiels élémentaires (tamis, canalisations, âtres, poulies...). C'est encore le cas au XVII siècle, mais, dans le sillage de Descartes, on rencontre alors également une thèse réductionniste radicale, selon laquelle les animaux ne sont littéralement que des machines, incapables de penser en général et en particulier de sentir 6 .

La charge de réalité des machines évoquées constitue la quatrième et dernière occasion de diversité. Certaines des machines décrites ou dessinées étaient réelles, les unes plus anciennes, les autres plus récentes; d'autres machines n'étaient pas impossibles technologiquement, mais non construites pour autant; d'autres enfin étaient manifestement irréalisables, ce qui ne les empêchait pas d'illustrer une idée, de constituer un argument pour une thèse ou de permettre une expérience de pensée. Dans ces conditions, certaines machines signifient sans se référer à des dispositifs réels ${ }^{7}$.

Étant donné la diversité des possibilités ainsi ouvertes par l'analogie entre machines et phénomènes naturels, on ne peut se contenter d'affirmations générales sur le rôle de l'idée de machine dans la Révolution scientifique ou sur ce que Dijksterhuis avait en son temps appelé la «mécanisation de la conception du monde ${ }^{8} »$. Le colloque « The Machine as Model and Metaphor » a donc rassemblé des historiens des techniques, de la science et de la philosophie soucieux d'explorer les présupposés de tous ordres liés à l'idée que les phénomènes naturels peuvent ou doivent être expliqués comme s'ils étaient des machines ou en tant que machines. Les différentes présentations se laissent rétrospectivement répartir en trois sessions; comme elles avaient été envoyées à l'avance aux conférenciers, beaucoup de temps a pu être consacré à la discussion.

L'objectif général de la première session, «Idea and Reality of Machines », était de cerner quelles espèces de dispositifs, existants ou imaginaires, étaient appréhendés comme des machines. Y a-t-il seulement quelque chose de commun entre des

6. Sur l'usage de comparaisons machiniques chez Galien, voir Ріснот, 1993, p. 129-222; BERRYMAN, 2002.

7. On a souvent fait deux reproches inverses aux théâtres de machines de la Renaissance: les machines représentées sont irréelles (voire irréalisables); elles ne contiennent pas d'innovation technique par rapport à celles qui existaient déjà. Pour une mise au point, voir Dolza et Vérin, 2003, qui conduit à une réflexion sur le genre littéraire du Théâtre de machines et à sa fonction dans ce que Marcus Popplow appelle une « culture de l'innovation ».

8. Dans l'ouvrage pionnier de DiJksterhuis, 1950, le terme « mécanisation » est employé pour des entreprises qui doivent être distinguées conceptuellement et historiquement. 
appareils de levage, des échafaudages, des engins de sièges, des mécanismes de transmission du mouvement, des véhicules, des horloges, des automates, des pompes, des moulins à eau et à vent, des instruments de mesure, des pendules, des dispositifs de mouvement perpétuel? À l'intérieur du genre constitué par les machines, les hommes de l'art de l'époque distinguaient d'ailleurs différentes espèces. Et sans doute faut-il introduire une autre espèce de distinction encore: parler des machines entre le XIII ${ }^{\mathrm{e}}$ et le $\mathrm{XVII}^{\mathrm{e}}$ siècle, voire au cours de la période longue qui s'étend de l'Antiquité jusqu'à l'époque moderne, cela suppose qu'on répertorie les innovations en matière de technique, d'organisation du travail ou de statut social qui purent modifier l'idée qu'on se faisait des machines.

Une question méthodologiquement importante dans ce contexte était d'identifier les sources historiques qui nous permettent d'appréhender ce qu'étaient ces fameuses machines. Contre une idée encore dominante historiographiquement, Giovanni di Pasquale, dans une présentation intitulée «Machines and Practical Knowledge in Antiquity ", soutint la thèse qu'en Grèce aussi bien qu'à Rome, et bien entendu particulièrement à l'âge d'or que fut la période hellénistique, les techniques étaient suffisamment développées pour constituer une technologie au sens fort du terme ${ }^{9}$. Cette thèse pose évidemment deux questions classiques, l'une de méthode - outre les admirables traités de Vitruve, de Héron d'Alexandrie, d'Apollodore de Damas et de Pappus d'Alexandrie, quelles sont les sources à notre disposition pour reconstituer le système technologique de l'Antiquité ? -, l'autre d'histoire générale. Si les techniques étaient suffisamment développées, et que les Anciens n'ont pas systématiquement dévalorisé les activités pratiques et utiles au profit d'un idéal de savoir théorique et désintéressé, que leur a-t-il donc manqué pour connaître une révolution technologique ou scientifique? La discussion qui a suivi cette présentation tendait à souligner l'importance d'une analyse pour ainsi dire quantitative de ce genre de phénomènes, qu'on pourrait présenter sous la forme conditionnelle suivante: même s'il n'y avait pas de différence d'essence ou de nature entre l'appréhension des machines chez les Anciens et chez les Modernes, il y aurait bien une différence, née de l'intensification des échanges intellectuels que permirent en particulier l'invention de l'imprimerie ou le développement des villes et des réseaux de communication.

La présentation qui suivait, «Idee und Wirklichkeit des Perpetuum mobile im Mittelalter » de Dietrich Lohrmann, s'est attachée à un dispositif idéel, le mouvement perpétuel, qui n'est pas sans incidence sur la pensée des machines ${ }^{10}$. Son enjeu historiographique était de rectifier l'idée selon laquelle la recherche de dispositifs de mouvement perpétuel n'aurait pas été possible avant la Renaissance, le Moyen Âge latin étant décrété trop enseveli dans des questions théoriques pour s'intéresser à un dispositif visant une économie d'énergie, et de toutes façons contraint à déclarer une telle entreprise impossible, étant donné l'adage aristotélicien « omne quod movetur $a b$

9. Pour un lecteur français, les références classiques sont sur ce point SCHUHL, 1938; KoYré, 1948. Pour une réhabilitation de la technologie hellénistique, voir cependant GILLE, 1980, et, de manière incontestablement outrée, Russo, 1996.

10. Cette présentation a été publiée, voir Lohrmann, 2006. Sur les mouvements perpétuels au XVII ${ }^{\mathrm{e}}$ siècle, voir GABBEY, 1985. 
alio movetur ». Il s'est agi de montrer que, ici comme ailleurs, la pensée scolastique est plus diversifiée qu'on ne le dit parfois, puis d'examiner des manuscrits d'ingénieurs du Moyen Âge tardif, en particulier ceux de l'allemand Konrad Gruter von Werden (1424) mais aussi ceux du vénitien Giovanni Fontana, qui présentent des perpetua mobilia et soulignent qu'aucun d'eux n'a à ce jour fonctionné ${ }^{11}$, de même que les critiques plus générales que Léonard de Vinci formule à propos de certains dispositifs. La discussion qui a suivi cette intervention a montré l'intérêt qu'il y aurait à faire l'histoire de ce rêve d'ingénieur, apparu en Inde au XII ${ }^{\mathrm{e}}$ siècle, condamné par l'Académie des sciences en 1775 et prouvé impossible en 1783 dans l'Essai sur les machines en général de Lazare Carnot. Il conviendrait en particulier d'analyser le contexte économique et intellectuel dans lequel la recherche de dispositifs de mouvement perpétuel s'insère, de montrer comment la conviction que cette recherche est vaine se développe en même temps que son intensification, de déterminer enfin ce qu'elle a apporté conceptuellement à la science mécanique.

La dernière présentation de cette session, celle de Marcus Popplow, «Idea and Reality of Machines in Early Modern Times », s'appuyait sur une base de données électronique rassemblant des dessins de machines du Moyen Âge tardif jusque dans les années 1650 pour analyser les différentes espèces de traces qui nous permettent d'appréhender les machines à la fois comme réalités et comme idées ${ }^{12}$. Dessins, modèles réduits, concours sur des projets spécifiques ou privilèges accordés pour des inventions, considérations théoriques et géométriques sur le fonctionnement des machines se développent et contribuent à constituer, dans le contexte d'une culture de l'innovation, un « discours», au sens foucaldien, sur les machines. Les quelques témoignages que nous avons de ce qu'étaient les pratiques quotidiennes des ingénieurs diffèrent cependant de ce « discours » sur les machines : ainsi, dans les carnets de notes de l'ingénieur allemand Heinrich Schickardt datant du début du XVII ${ }^{\mathrm{e}}$ siècle, on trouve des machines différant à plusieurs égards de celles que l'on trouve dans les Théâtres de machines: il s'agit de machines plus simples, dessinées avec des conventions graphiques différentes, complétées de mesure et d'explications verbales. Sans doute ne faut-il pas se hâter de conclure que ces carnets de notes, au demeurant rares, représentent plus fidèlement les machines que des ouvrages publiés dans le cadre d'une culture de l'innovation: du moins permettent-ils de montrer que, par-delà le discours sur les machines, la question de la reconstitution des machines est vive.

La session «Machines Beyond Mechanics» étudiait l'extension problématique de la référence aux machines dans différents domaines scientifiques. Le problème était d'examiner les raisons de cette extension, mais aussi de prendre la mesure de la diversité des usages de la notion de machine selon les domaines considérés. Une des thèses qui peut être tenue pour caractéristique de ce que l'on appelle usuellement la « philosophie mécanique » ou le « mécanisme » est par exemple que tout est machine.

11. Les manuscrits ont été récemment édités, traduits et dûment commentés dans GRUTER VON WERDEN, 2006.

12. Cette base de données, réalisée par Marcus Popplow et Wolfgang Lefèvre dans le cadre d'un projet du Max-Planck-Institut für Wissenschaftsgeschichte, peut être consultée à l'adresse suivante: http://dmd.mpiwg-berlin.mpg.de/home. Elle est présentée dans Lefèvre et Popplow, 2006. Sur les problèmes posés par l'analyse historique des dessins de machines, voir également LEFÈVRE, 2004. 
Mais, étant donné l'équivocité de la notion de machine rappelée en introduction puis dans les paragraphes consacrés à la première session de ce colloque, il est clair que cette thèse avait pour destin de se diversifier selon les besoins de la cause.

La présentation de Jochen Büttner, «Mental Models Between Practical and Theoretical Knowledge. The Example of Impact » avait une double ambition : il s'agissait à la fois d'analyser les obstacles épistémologiques auxquels s'est heurtée la compréhension du phénomène de la percussion et de montrer que la notion de modèle mental est particulièrement adaptée pour décrire les interactions entre le savoir pratique qui est celui des hommes de l'art et le savoir mis en forme dans des traités argumentatifs et déductifs ${ }^{13}$. On pourrait se demander quel est le rapport entre le phénomène de la percussion et la question des machines: en fait, celui-ci est tout à fait étroit jusqu'au $\mathrm{XVII}^{\mathrm{e}}$ siècle, au moins pour deux raisons. D'une part, selon les écrits du pseudo-Aristote, de Héron ou de Pappus, appelant « machine » tout ce qui permet d'augmenter une force et de produire de grands effets avec une petite puissance, eu égard à ses effets, la percussion est une machine: un poids agit plus lorsqu'il percute un corps que lorsqu'il est posé sur lui. D'autre part, et de manière plus importante, jusqu'à la fin du XVIII ${ }^{e}$ siècle, on tente de comprendre le phénomène de la percussion comme un phénomène statique, autrement dit de chercher à identifier les longueurs et les poids qui permettraient d'appliquer le principe du levier qui est le principe auquel sont réduits les machines simples. Bien évidemment, il y a là un cas où le modèle des machines a finalement constitué un obstacle, la percussion et le poids n'étant pas des grandeurs physiques de même dimension. La présentation de Sven Dupré, «Celestial Machines, Hyperbolic Lenses and Method » était moins facile à évaluer du point de vue d'une épistémologie récurrente: il s'agissait non seulement d'examiner la réception anglaise de la machine à polir des lentilles hyperboliques qui constituait la finalité proprement technique de la Dioptrique de Descartes, mais aussi de souligner l'analogie que certains textes de Bacon et de Descartes établissent entre le travail implacablement produit par une machine et les promesses d'une méthode dont tous pourraient faire usage, indépendamment de leurs qualités propres. Là encore, les machines n'étaient pas là où on les attendait.

Les présentations de Christoph Lüthy et de Sophie Roux, sans toujours partir des mêmes sources, étaient animées d'une même inquiétude. À force de circuler comme un emblème de main en main, la machine n'est-elle pas, de modèle devenue métaphore, et de métaphore vive devenue métaphore morte? Christoph Lüthy, dans « Micro-machines Explaining the World », partait du célèbre passage de la préface de Some Specimens of an Attempt to Make Chemical Experiments Useful to Illustrate the Notions of the Corpuscular Philosophy dans lequel on voit usuellement le certificat de naissance de la philosophie mécanique. Dans ce passage, Boyle présente comme équivalentes la philosophie mécanique, d'après laquelle tous les phénomènes naturels peuvent être compris par des engins mécaniques, et la philosophie corpusculaire, d'après laquelle ils sont

13. La présentation la plus explicite de la notion de modèle mental ici mise en œuvre est celle de Damerow et Renn, 2006. Sur le problème de la percussion, voir également Bertoloni Meli, 2006, p. 140-144, p. 227-239; et Sophie Roux, " Quelles mathématiques pour la force de percussion?», dans Rommevaux (Sabine), dir., Formes d'interaction entre mathématiques et philosophie naturelle, Actes du colloque international, Tours, novembre 2007, à paraître. 
réductibles à des corpuscules. Mais, remarquait Lüthy, ce que l'on trouve en fait dans bien des textes, ce sont des fluides en mouvement et des corpuscules pleins de vie. Dès lors, s'intéressant aux « micro-machines » composant les corps vivants, il put montrer que les anatomistes qui succédèrent à Descartes furent confrontés au dilemme suivant: ou bien, comme Theodor Craanen, être fidèles à l'inspiration qui avait été celle de Descartes, mais perdre alors tout contact avec les réalités anatomiques; ou bien, comme Giovanni Alfonso Borelli et ses jeunes disciples (Marcello Malpighi, Lorenzo Bellini, Carlo Fracassati), être fidèles à ce qu'ils voyaient dans leurs microscopes, mais alors, définitivement abandonner le paradigme cartésien ${ }^{14}$.

Dans le même esprit, la présentation de Sophie Roux, « Corpuscularisme, mécanisme, machinisme. Pourquoi la métaphore de la machine dans la philosophie mécanique?» proposa pour commencer de distinguer plus fermement qu'on ne le fait d'ordinaire trois thèses: le corpuscularisme, selon lequel les phénomènes naturels sont ultimement réductibles à des corpuscules; le mécanisme, qui est une restriction du précédent, selon laquelle les processus naturels résultent des lois du mouvement appliquées à des corpuscules inertes; le machinisme enfin, qui consiste à comparer les phénomènes à des machines. Or, si le mécanisme est une thèse forte, et par là-même contraignante, il n'en est pas de même pour le corpuscularisme, qui peut être concilié avec toutes sortes de philosophies de la nature, en particulier selon les propriétés que l'on attribue aux corpuscules et selon le type d'interaction que l'on suppose entre eux. Dès lors, la question qui se pose est d'identifier les raisons pour lesquelles bien des philosophes naturels ne se sont pas satisfaits du corpuscularisme, mais ont recouru au machinisme. Autrement dit, quels sont les avantages intellectuels de la thèse que tout est machine par rapport à la thèse que tout est fait de corpuscules? La réponse à cette question suppose qu'on distingue le cas des animaux et le cas du monde, qui sont les deux réalités à propos desquelles la comparaison avec des machines revient le plus souvent.

La troisième et dernière session était justement consacrée à l'analyse détaillée du monde comme machine. Depuis l'Antiquité, le terme «machina» est utilisé métaphoriquement à propos du monde, mais la dénotation de l'expression « machina mundi » change cependant au cours de la Renaissance ${ }^{15}$. Dans l'Antiquité et au Moyen Âge, elle se rapportait au monde visible, conçu comme un édifice stable composé des terres, des mers et des cieux, ou bien, si l'on veut, des éléments sublunaires et de l'élément supralunaire. À partir de la Renaissance, elle se rapporte à une machine qui transmet du mouvement et dont les principes cachés doivent être mis au jour par la science mécanique. L'idée que les mouvements cosmiques peuvent être analysés selon les mêmes principes que les phénomènes sublunaires, et que ces principes se laissent voir dans le fonctionnement des machines, semble également nouvelle. C'est aussi l'époque où l'image plus spécifique de l'horloge du monde, pour la première fois employée

14. L'originalité de cette présentation était d'étudier les structures révélées par le microscope; quant à la comparaison "macroscopique » entre organismes vivants et automates, elle a déjà été l'objet d'innombrables études, parmi lesquelles on retiendra, à un titre ou à un autre: CANGUILHEM, 1952; Des Chene, 2001 ; Galluzzi, 1987.

15. Je résume ici à gros traits Baroncini, 1989; McLaughlin, 1994; Popplow, 1998, p. 95-115; Popplow, 2007, p. 48-60. 
par Oresme, devient un lieu commun ${ }^{16}$. Les questions examinées dans cette session étaient dès lors de déterminer les modalités et les conséquences de l'idée que l'univers était une machine, mais aussi les alternatives à cette idée ${ }^{17}$. Une fois encore, il fallut constater la plurivocité de la machine.

La présentation d'Elly Truit, « Microcosm and Macrocosm. Automata and Clockwork in Medieval Europe » examinait la signification dans la culture médiévale latine de deux espèces de machines : l'horloge, qui représente usuellement le monde, et les automates, qui représentent les animaux ${ }^{18}$. Jusqu'au XIV ${ }^{\mathrm{e}}$ siècle, ces machines complexes existent principalement à titre de présents venus de l'Orient ou de fantaisies littéraires ; mais ensuite, elles apparaissent bien dans l'Europe médiévale comme des produits artificiels du travail humain, capables de donner des indications sur ce que sont les phénomènes naturels : c'est en particulier le cas de la grande horloge de Strasbourg, magnifiquement reconstruite par Conrad Dasydopius dans la seconde moitié du $\mathrm{XvI}^{\mathrm{e}}$ siècle. Deux présentations suivaient deux des pistes ouvertes par cet exposé.

Dans « Roughing up the Elements : raptus and the Celestial-Terrestial Boundary », Michael Shank analysa le problème mécanique que pose, dans la cosmologie aristotélicienne, la co-existence à la frontière du sublunaire et du supralunaire de mouvements rectilignes et circulaires. Suivant à la trace la notion de raptus et la théorie des marées, il montra que le problème émergea chez Aristote, puis fut ensuite développé chez Simplicius, al-Bitruji, Francesco Capuano de Manfredonia, Alessandro Achillini, tant et si bien que l'on en trouve des traces, encore inexpliquées, chez Francis Bacon et Galilée. Comme dans la présentation précédente, on voyait ici les limites de la périodisation usuelle: un problème conceptuel persiste à travers différents changements de paradigme. Un point important, sur lequel on reviendra en conclusion, était que l'on trouve chez des auteurs médiévaux une manière mécanique d'analyser certains problèmes: le supralunaire a beau être un lieu d'immuabilité, la plausibilité d'un système du monde suppose que ses conditions de possibilités mécaniques aient été explorées ${ }^{19}$.

Le point de départ de la présentation de Peter McLaughlin, «The World as an Autonomous Mechanism. Descartes and his Successors » était l'évolution sémantique au terme de laquelle l'expression «machina mundi» en vint à se rapporter principalement à ce qui a été appelé plus haut des «machines marxistes ». Mais, remarquait McLaughlin, le monde est une « machine marxiste » paradoxale, qui ne produit aucun travail: comme l'horloge, elle se contente de transmettre du mouvement, sans perte, mais aussi sans gain. Assez classiquement, furent ensuite examinées les contraintes conceptuelles imposées par la comparaison du monde à une horloge, en particulier en termes de lois de conservation, mais aussi les différences que cette comparaison

16. Sur l'image de l'horloge, voir MAYr, 1986. Popplow, 2007, p. 65-66, rappelle que, contrairement à ses successeurs, Oresme ne voit pas l'horloge comme un système de transmission du mouvement.

17. Sur l'interaction entre cosmologie et mécanique, voir également BERTOLONI MeLI, 2006, p. 190-223; Bucciantini, Camerota et Roux, dir., 2007.

18. L'éminence de ces deux espèces de machines avait déjà été dégagée dans l'article pionnier de PRICE, 1964.

19. Cette idée avait déjà été développée à propos d'autres exemples dans un autre colloque, voir SHANK, 2007. 
autorisait - par exemple en ce qui concerne les grandeurs qui sont conservées, mais aussi les modalités des lois de conservation.

La dernière présentation enfin, celle de Rivka Feldhay, « The Earth Lifting Machine. Negociating Cultural and Scientific Boundaries ", portait sur la curieuse interaction chez des jésuites du XVII ${ }^{\mathrm{e}}$ siècle, Christoph Grienberger, Paolo Casati, Gaspar Schott et Paul Guldin, de la cosmologie et de la mécanique entendue comme théorie des machines simples. Tous cherchèrent en effet à calculer, selon le mot d'Archimède que l'on citera ici dans leur langue, «da mihi ubi consistam et terram movebo », les dimensions d'une machine qui serait susceptible de soulever la Terre. Comme souvent dans la science jésuite, cette curieuse entreprise demande à être interprétée en termes de revendications disciplinaires.

Pour conclure, on commencera par rappeler quelques évidences. En premier lieu, l'appréhension du machinisme en général, à quelque époque que ce soit, ne peut faire l'économie d'une recherche interdisciplinaire, rassemblant des historiens des techniques, des sciences et de la philosophie, de la littérature enfin. En second lieu, elle a tout à gagner d'un travail comparatif sur de longues périodes : le colloque «The Machine as Model and Metaphor » était organisé dans le cadre d'un programme centré sur la grandeur et la décadence des universités aristotéliciennes; continuellement, nous avons été renvoyés à l'Antiquité ou au Moyen Âge; régulièrement aussi, nous avons déploré le peu de travaux qui existent sur les machines et la science mécanique arabes et islamiques ${ }^{20}$. À cet égard, les bases de données mises en place par le Max-Planck-Institut für Wissenschaftsgeschichte sont évidemment décisives, et elles devraient non seulement faciliter notre travail, mais aussi modifier notre recherche ${ }^{21}$.

Mais peut-être aussi faut-il indiquer que cette ouverture disciplinaire et historique ne sera profitable qu'à condition de se donner les moyens d'articuler effectivement des univers qui risquent sans cela d'apparaître comme autant de mondes parallèles, rapprochés seulement le temps d'une rencontre académique. Il sera ici crucial de cerner, historiquement aussi bien que conceptuellement, ce que l'on pourrait appeler le mode de pensée mécanique des «praticiens ${ }^{22}$. En parlant de «praticiens », on pense ici aux ouvriers, artisans, hommes de l'art et ingénieurs, qui pouvaient éventuellement mettre la main à la pâte, construire des modèles de machines et en dessiner, et qui, en tout état de cause, manipulaient des instruments, projetaient des constructions, bref, contribuaient à l'élaboration de machines en tous genres. Par «mode de pensée mécanique », on entend les activités mentales qui correspondent aux opérations fondamentales de ces praticiens: évaluer les grandeurs ou mesurer, faire des esquisses et des modèles, choisir des matériaux

20. Pour mémoire, si un des volumes issus de ce programme (LAIRD et Roux, dir., 2008) ne comprend pas de présentation consacrée exclusivement aux rapports entre mécanique et philosophie naturelle dans les savoirs arabes et islamiques, c'est que le conférencier invité à cette occasion, Mohammed Abattouy, n'avait pu obtenu de visa pour Tenerife où se tenait le colloque, et n'a par suite pas souhaité contribuer au volume.

21.Outre la base de données indiquée supra note 12, voir le projet Archimedes : http://archimedes2. mpiwg-berlin.mpg.de/archimedes_templates.

22. À ma connaissance, il n'y a pas de recherches systématiques sur ce point, mais on trouve des jalons pour une réflexion à venir dans BenNett, 1980, 1986, 1998; BERRYMAn, 2002 et 2003 ; Bertoloni Meli, 2006; Vérin, 1993 ; Vérin et Dubourg Glatigny, dir., 2008. 
adéquats, ajuster des parties mouvantes, tenir compte des frottements, évaluer les forces disponibles, les combiner pour maximiser le gain, etc. La question plus historique sera de définir dans quelle mesure l'évolution des techniques et des sciences modifie ces opérations fondamentales. La question plus conceptuelle consistera à déterminer comment il convient d'appréhender des « pratiques », des « savoirs tacites », des « savoir-faire » qui ne sont pas formalisés, et dans certains cas, pas même explicites. Comme tout colloque qui se respecte, le colloque « The Machine as Model and Metaphor » aura donc répondu à quelques questions, mais en aura surtout ouvertes de nouvelles.

\section{LISTE DES RÉFÉRENCES}

BARONCINI (Gabriele), 1989, "Note sulla formazione del lessico della metafora "machina mundi" », Nuncius. Annali di storia della scienza, vol. 4, n 2, p. 3-30.

Bennet (Jim), 1980, « Robert Hooke as Mechanic and Natural Philosopher », Notes and Records of the Royal Society of London, ${ }^{\circ} 35$, p. 33-48.

Bennet (J.), 1986, «The Mechanics Philosophy and the Mechanical Philosophy », History of Science, $\mathrm{n}^{\circ} 24$, p. 1-28.

Bennet (J.), 1998, «Practical Geometry and Operative Knowledge », Configurations, n 6 , p. $195-222$.

Berryman (Sylvia), 2002, "Galen and the Mechanical Philosophy », Apeiron. A Journal for Ancient Philosophy and Science, ${ }^{\circ}$ 35, p. 85-97.

Berryman (S.), 2003, « Ancient Automata and Mechanical Explanation », Phronesis, vol. XLVIII, $\mathrm{n}^{\circ} 4$, p. 344-369.

Bertoloni Meli (Domenico), 2006, Thinking with Objects. The Transformation of Mechanics in the Seventeenth Century, Baltimore, Johns Hopkins University Press.

Bucciantini (Massimo), Camerota (Michele) et Roux (Sophie), dir., 2007, Mechanics and Cosmology in the Medieval and Early Modern Period, Florence, Olschki (Nuncius).

CAnguilhem (Georges), 1952, « Machine et organisme », rééd. dans La Connaissance de la vie, Paris, Vrin, 1989, p. 124-159.

Damerow (Peter) et RenN (Jürgen), 2006, « Mentale Modelle als kognitive Instrumente der Transformation von technischem Wissen », dans RenN (Jürgen) et al., dir., Weight, Motion and Force. Conceptual Structural Changes in Ancient Knowledge as a Result of its Transmission, Berlin, Max-Planck-Institut für Wissenschaftsgeschichte (Preprint 320), p. 4-25.

Des Chene (Dennis), 2001, Spirits and Clocks. Machine and Organism in Descartes, Ithaca, Cornell University Press.

Dijksterhuis (Eduard Jan), 1950, The Mechanization of the World Picture, trad. angl. Carry Dikshoorn, Oxford, Clarendon Press, 1961.

Dolza (Luisa) et Vérin (Hélène), 2003, «Une mise en scène de la technique. Les Théâtres de machines », Alliage, ${ }^{\circ}$ 50-51, p. 8-20.

Gabbey (Alan), 1985, «The Mechanical Philosophy and its Problems. Mechanical Explanations, Impenetrability and Perpetual Motion », dans PIтT (Joseph C.), dir., Change and Progress in Modern Science, Dortrecht, Reidel, p. 9-84.

Galluzzi (Paolo), 1987, «Leonardo da Vinci. From the "elementali macchinali" to the manmachine », History and Technology, vol. 4, n 4, p. 235-265.

GiLle (Bertrand), 1980, Les Mécaniciens grecs, Paris, Le Seuil (Science ouverte). 
Gruter von Werden (Konrad), 2006, De machinis et rebus mechanicis. Ein Maschinenbuch aus Italien für den König von Dänemark 1393-1424, éd. Dietrich LoHrmanN, Horst KRANZ et Ulrich AlerTz, Città del Vaticano, Bibliotheca Apostolica Vaticana (Studi e testi), 2 vol.

KOYRÉ (Alexandre), 1948, « Les philosophes et la machine », rééd. dans Études d'histoire de la pensée philosophique, Paris, Gallimard, 1971, p. 305-340.

LAIRD (Walter Roy) et Roux (Sophie), dir., 2008, Mechanics and Natural Philosophy before the Scientific Revolution, Dordrecht, Springer (Boston Studies in the Philosophy of Science).

LefĖvre (Wolfgang), 2004, Picturing Machines 1400-1700, Cambridge, MIT Press.

LefĖvre (Wolfgang) et Popplow (Marcus), 2006, « Database machine drawings. Ein Instrument zur Erschließung und Erforschung der Maschinenzeichnungen der Renaissance-Ingenieure », Technikgeschichte, vol. LXXIII, ${ }^{\circ}$ 2, p. 115-120.

Lohrmann (Dietrich), 2006, «Idee und Wirklichkeit des Perpetuum mobile im Mittelalter», Technikgeschichte, vol. LXXIII, $n^{\circ} 3-4$, p. 227-252.

Marx (Karl), 1867, Le Capital, rééd. dans Euvres de Karl Marx, éd. Maximilien Rubel, Paris, Gallimard (La Pléiade), 1965, 2 vol.

MAYr (Otto), 1986, Authority, Liberty and Automatic Machinery in Early Modern Europe, Baltimore/Londres, Johns Hopkins University Press.

McLaughlin (Peter), 1994, «Die Welt als Maschine. Zur Genese des neuzeitlichen Maschinenbegriffs », dans Grote (Andreas), dir., Macrocosmos in Microcosmos. Die Welt in der Stube. Zur Geschichte des Sammels 1450 bis 1800, Opladen, Leske et Budrich, p. 439-451.

Ріснот (André), 1993, Histoire de la notion de vie, Paris, Gallimard.

Popplow (Marcus), 1998, Neu, nützlich und Erfindungsreich. Die Idealisierung von Technik in der frühen Neuzeit, Münster/New York/München/Berlin, Waxmann (Cottbuser Studien zur Geschichte von Technik, Arbeit und Umwelt).

Popplow (M.), 2007, « Setting the World Machine in Motion. The Meaning of Machina Mundi in the Middle Ages and the Early Modern Period», dans Bucciantini (Massimo), CAmerota (Michele) et Roux (Sophie), dir., Mechanics and Cosmology in the Medieval and Early Modern Period, Florence, Olschki (Nuncius), p. 45-70.

Price (Derek J. de Solla), 1964, «Automata and the Origins of Mechanism and Mechanistic Philosophy », Technology and Culture, vol. V, p. 9-23.

Russo (Lucio), 1996, The Forgotten Revolution. How Science Was Born in 300 BC and Why it Had to Be Reborn, trad. am. Silvio Levy, Berlin/Heidelberg/New York, Springer Verlag, 2004.

SChlanger (Judith), 1971, Les Métaphores de l'organisme, rééd. Paris, L'Harmattan (Histoire des Sciences Humaines), 1995.

Schunl (Pierre-Maxime), 1938, Machinisme et philosophie, rééd. Paris, Presses universitaires de France, 1947.

SHAnk (Michael), 2007, « Mechanical Thinking in European Astronomy », dans BucciantinI (Massimo), Camerota (Michele) et Roux (Sophie), dir., Mechanics and Cosmology in the Medieval and Early Modern Period, Florence, Olschki (Nuncius), p. 3-28.

Veneziani (Marco), 2005, Machina. XI Colloquio internazionale (Roma, 8-10 gennaio 2004), Florence, Olschki (Lessico intellettuale europeo).

VÉRIN (Hélène), 1993, La Gloire des ingénieurs. L'intelligence technique du XVI au XVIII siècle, Paris, Albin Michel.

Vérin (Hélène) et Dubourg Glatigny (Pascal), dir., 2008, Réduire en art. La technologie de la Renaissance aux Lumières, Paris, Éditions de la Maison des sciences de l'homme. 